\title{
Live Fast, Die Young: New Lessons in Mammalian Longevity
}

\author{
Lynne S. Cox
}

\begin{abstract}
Can we extend human lifespan? Do we need to regulate lifestyle choices or can we simply pop a pill to make us live longer? These are questions raised by two new studies demonstrating significant lifespan extension in mice fed the drug rapamycin in their diet and in calorically restricted rhesus monkeys. The excitement generated by these papers is a consequence of their novelty and possible applicability to humans: the mouse study on rapamycin is the first to highlight a drug that given in the diet late in life leads to increased mammalian longevity, and the Wisconsin monkey study is the first to report significant lifespan extension and delay in ageassociated morbidity in nonhuman primates following adult-onset caloric restriction. These papers are discussed here in the context of current knowledge of molecular mechanisms of aging.
\end{abstract}

\section{Lifespan modulation strategies in mammals}

I NCREASES IN MAMMALIAN LIFESPAN are seen as the gold-standard test of aging interventions relevant to human health and longevity. Two new articles describe significant increases in the lifespan of mice fed dietary rapamycin, ${ }^{1}$ and in rhesus monkeys subjected to adult-onset caloric restrictions. ${ }^{2}$ The mouse study ${ }^{1}$ was conducted as part of the National Institute on Aging (NIA) Intervention Testing Program (ITP) across three sites in the United States-The Jackson Laboratory (TJL), the University of Michigan (UM), and the University of Texas Health Science Centre (UT) — and by using a total of 1960 mice at three independent test facilities, it is a statistically powerful study. The findings that make this study particularly interesting are that a clinically licensed drug given late in life can significantly extend lifespan in genetically heterogeneous mammals. Thus, genetic factors contributing either to longevity or drug response were, to an extent, ruled out. Rapamycin administration in the diet from 600 days of age (which the authors equate to 60 years of human life) showed both that an antiaging drug can be given orally and that such drug therapy can be started in an aged population with clear longevity benefits. As a note of extreme caution, rapamycin is a potent immunosuppressive agent, used clinically to prevent transplant rejection, and has serious side effects that would not be acceptable in an otherwise healthy human patient. The mice were not adversely affected by immune suppression because they were kept in a pathogen-free environment where they were not exposed to infectious agents.

In the Wisconsin study led by Rick Weindruch, ${ }^{2}$ a cohort of 30 male rhesus monkeys (15 controls and 15 calorie re- stricted [CR]) was established 20 years ago in 1989, and added to in 1994 with 30 females and a further 16 males. Restriction by $30 \%$ of the animal's individual ad libitum daily food intake was introduced over a 3-month period in adult monkeys. At the current stage in the study (July, 2009), half of the control animals are alive whereas $80 \%$ of the CR monkeys have survived, a statistically significant $(p=0.03)$ increase in survival in CR monkeys. On multiple measures of health (glucose regulation, endocrine profiles, blood pressure, electrocardiogram [ECG], brain magnetic resonance imaging [MRI], X-ray absorptiometry of lean muscle), the CR monkeys are healthier than controls, showing also lower incidence of cancer and cardiovascular disease.

\section{Analysis of study design: rapamycin feeding to mice}

The original design of the ITP protocol was to administer the drug to the mice in diet at 120 days of age. Initial problems with rapamycin dosing led to development of a stable encapsulated form of rapamycin suitable for dietary administration that was enterically coated to ensure absorption in the lower intestine and to achieve appropriately high blood concentrations of the drug. Because of the time taken to develop this form of rapamycin, the drug treatment took place in old mice starting from 600 days; other interventions tested in parallel mouse cohorts began at only 120 days. However, this delay in rapamycin treatment due to technical problems was fortuitous because it revealed for the first time that a small-molecule intervention could be active in extending lifespan, even when administered to older animals. An additional study where rapamycin administration began much earlier (at

Department of Biochemistry, University of Oxford, Oxford, United Kingdom. 
270 days) is also in progress, and data presented ${ }^{1}$ suggest that $^{2}$ this too enhances longevity in mice. This further verifies that rapamycin is acting through the taget of rapamycin (TOR) kinases signaling pathway because phosphorylation of a downstream target of TOR, ribosomal S6 kinase (see Fig. 1 and below), was severely abrogated in visceral fat pads of mice treated with rapamycin compared with controls.

The ITP study on rapamycin feeding to mice avoided the possibility that any observed lifespan extension was simply a consequence of a specific genetic trait in a particular laboratory mouse strain, because the mice tested were the offspring of CB6F1 females and C3D2F1 males, used specifically to generate heterogeneity. Although these mice were obviously not genetically pure like standard inbred strains, it is not clear whether full siblings represent a truly heterogeneous population.

How significant were the lifespan extensions in the rapamycin-fed mice? Overall, taking the data from all three sites together, age at $90 \%$ mortality was $14 \%$ higher for female mice and $9 \%$ higher for males than the controls. ${ }^{1}$ When the data are analyzed by site, however, it is apparent that male mice at both UM and UT destined for rapamycin feeding were already showing lifespan extension, even before the drug was administered.

Given the well-characterized impact of weaning and early life diet on lifespan, ${ }^{3}$ it is unfortunate that the cohorts of mice in the control group (or subject to Enalapril or caffeic acid phenylethyl ester feeding interventions) were fed on Purina 5LG6 from 120 days, while the groups at UM and UT that would be given rapamycin were fed on Purina 5008 (UM) or Teklad 7912 (UT) up until the point at which rapamycin was introduced at 600 days, when the diet was shifted to Purina 5LG6. (Note that that mice at The Jackson Laboratory were maintained on Purina 5LG6, the same diet as used for rapamycin feeding, from weaning onward). This may seem a somewhat esoteric point, but in addition to possible differences in lifelong physiology according to earlylife diet, ${ }^{3}$ changes in mouse food can lead to transient changes in body weight, ${ }^{4}$ and body weight fluctuations may also have an impact on health and longevity. However, at

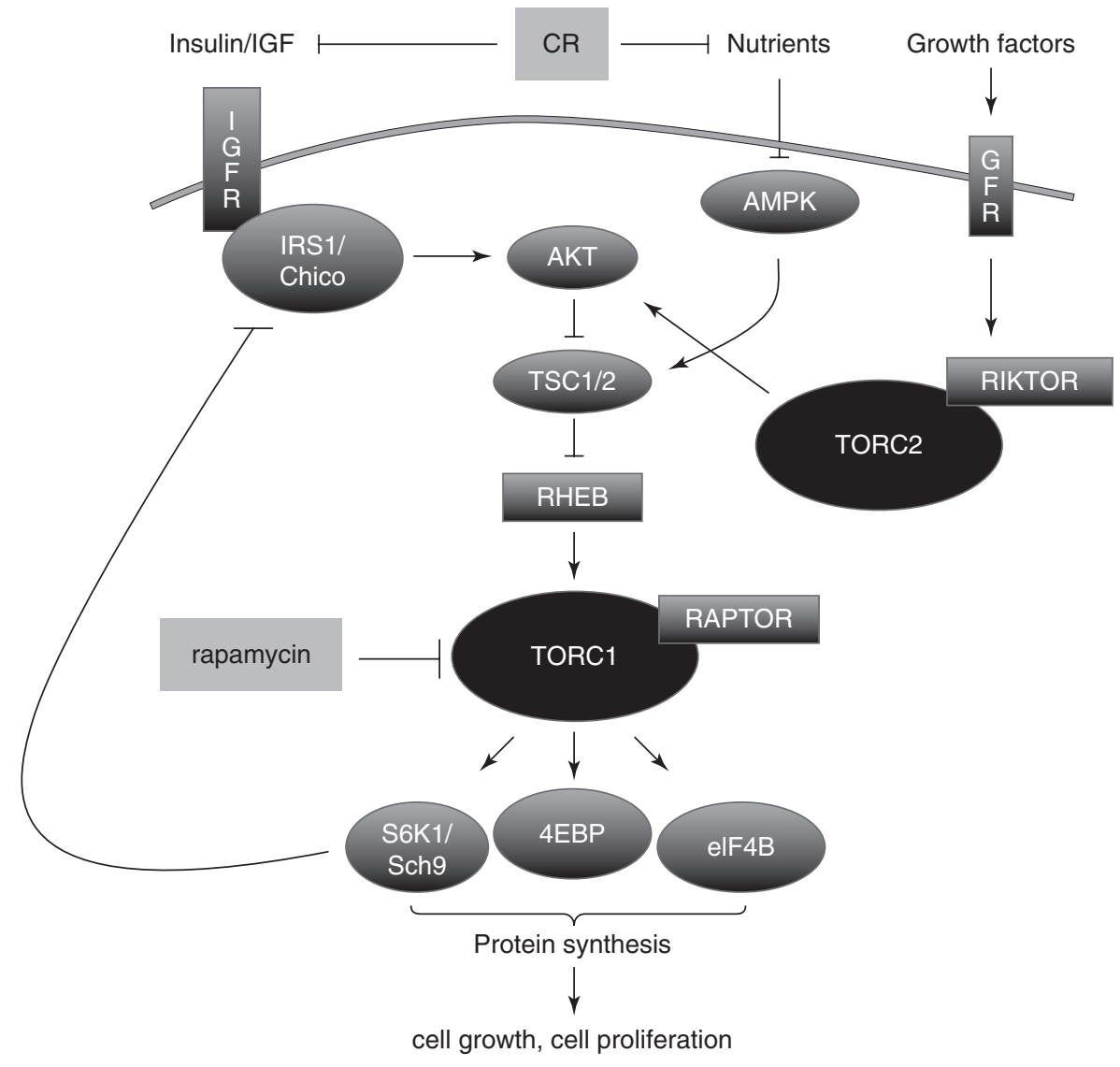

FIG. 1. Impact or rapamycin and calorie restriction (CR) on target of rapamycin (TOR) signaling. A highly simplified pathway is shown, leading from insulin or insulin-like growth factor (IGF1) through the transmembrane IGF receptor and via the insulin receptor substrate (chico in flies) to the AKT kinase. Activated AKT kinase exerts an inhibitory effect on the Tuberous Sclerosis Complex (TSC1/2), which would otherwise inhibit a TOR activator, RHEB (Ras homolog enriched in brain). Thus, insulin signaling activated TOR kinase. Downstream effectors of TOR are the ribosomal S6 kinase (Sch9 in yeast) together with translation initiation factor 4 binding protein (4EBP) and eukaryotic translation initiation factor 4B (eIF4B). Activation of these components leads to increased protein synthesis with cell growth and proliferation. Thus, rapamycin inhibition of TOR signaling leads to a decrease in protein synthesis and is thought to shift cells from a proliferative mode to a maintenance and repair mode. CR leads to decreases in insulin signaling and so may impact indirectly on TOR signaling. It is also possible that reduced nutrient levels also activate adenosine monophosphate (AMP) kinase, result ing in TOR inhibition by TSC1/2. IGFR, Insulin growth factor receptor; AMPK, AMP-activated protein kinase; GFR, growth factor receptor. (For color version of image, see: www.liebertpub.com/rej). 
TJL where the core diet was maintained constant throughout, the lifespan extension for females (17\%) was greater than at the other two sites; for males, TJL mice on rapamycin showed a greater percentage increase in age $(10 \%)$ at the $90 \%$ percentile than those at UM, though much smaller increases than at UT.

Even if only the data from TJL are examined (since the controls are true controls), the observed lifespan extensions on rapamycin feeding from 600 days are highly significant. Thus, it appears that the feeding issues have not adversely affected the outcome of the experiment, and that the pooled data for lifespan extension across all three sites provides a reasonable measure of the effect of rapamycin on longevity.

The doses used in mice to obtain the $9-14 \%$ extension in lifespan were very high: $2.24 \mathrm{mg}$ of rapamycin per $\mathrm{kg}$ of body weight per day, compared with a standard human immunosuppressive dose of 2-5 mg/day (approximately 40 $\mu \mathrm{g} / \mathrm{kg}$ per day). This dosing is very high when also compared with that used experimentally in other rodents, e.g., a dose of $0.5 \mathrm{mg} / \mathrm{kg}$ per day was sufficient to inhibit TOR-mediated liver fibrosis and improve both liver function and survival in cirrhotic rats. ${ }^{5}$ A further ITP-type study testing the impact of different rapamycin doses on longevity would be very interesting because it is plausible that lower doses may maintain longevity benefits with fewer side effects.

\section{Do interventions that increase longevity increase health span?}

In the mouse rapamycin study by Harrison et al., ${ }^{1}$ where causes of death were ascertained by necropsy, there appeared to be little difference in age-associated diseases as causes of death in 40 of the rapamycin-fed mice compared with 31 control mice, although a higher incidence of cancer (34/40, 85\% rapamycin vs. $23 / 31,74 \%$ controls) and cardiovascular disease $(5 / 40,12.5 \%$ rapamycin vs. $2 / 31,6 \%$ controls) is of concern. Interestingly, more of the control group succumbed to inflammation or possible infection (abscesses and septicemia) than the rapamycin group, although numbers are too small to derive significance. By contrast, over the 20 years so far of the calorie restriction study in rhesus monkeys, Colman et al. ${ }^{2}$ report both subjective improvements (CR monkeys appeared younger than controls) and objective delay in onset-of-age-related pathologies including sarcopenia, ${ }^{6}$ atrophy of grey matter in the brain, cancer incidence (8 cases in controls and only 4 in CR monkeys), and diabetes (none in CR group compared with 5 diabetic and 11 prediabetic in the control group). To date, whereas around four fifths of control monkeys presented with age-related disease, only one third of the CR group were affected. ${ }^{2}$ This is a highly significant result $(p=0.008)$, with a hazard ratio of 2.9, and it strongly implies that the CR monkeys are biologically younger than the controls.

These findings are consistent with earlier studies on rodents, where various age-associated pathologies were reduced or delayed on CR diets (e.g., see ref. 7). Studies on human volunteers (especially from the Caloric Restriction Society) have also reported significant health improvement following calorie restriction, with very marked decreases in low-density lipoprotein (LDL) and triglyceride levels, increased high-density lipoprotein (HDL), reduced blood pressure, and low plasma insulin and fasting plasma glucose concentrations (e.g., refs. 8 and 9; reviewed in ref. 10).
What the latest study by the Weindruch group ${ }^{2}$ adds to this are data on lifespan, suggesting a significant increase in longevity of CR rhesus monkeys, and physical evidence of retention of grey matter in the brain. Thus, a "quick-fix" antiaging pill based on rapamycin may increase longevity but may not be able to reduce the morbidity of old age, whereas calorie restriction certainly seems powerful in delaying onset of disease, although with the caution that bone mineral density may be decreased on calorie restriction in humans. ${ }^{11}$ It is worth bearing in mind that hip fractures are a major cause of morbidity and mortality in older people (e.g., refs. 12 and 13), so any lifespan gains obtained by calorie restriction may be abruptly removed by the adverse consequences of low bone density. The rhesus monkeys did not show any fractures; however, anatomical differences in hip structure and bipedal gait may make human hips more prone to fracture. It is also likely that the monkeys were not exposed to the same trip hazards as elderly humans living independently.

Will it be possible to obtain the lifespan extension observed in both CR monkeys and rapamycin mice without the long-term need for calorie restriction or the risks of serious side effects with rapamycin? Aiming to increase the health span, i.e., the period of life free of age-related disease, appears to me a reasonable goal and far preferable to lifespan extension at all costs. To assess the possibility of developing such an intervention, it is necessary to understand the molecular pathways that calorie restriction and rapamycin act on to increase lifespan.

\section{Insulin signaling and rapamycin inhibition converge on the TOR signaling pathway}

Calorie restriction (also dietary restriction, or DR) without malnutrition has already been shown to increase lifespan in invertebrate model systems and in mammals (reviewed in ref. 14). Is the absence of nutrients the converse of high levels of nutrient availability? If so, calorie restriction should act through the insulin/insulin growth factor1 (IGF1) signaling (IIS) pathway used by the cell to signal nutrient availability. Lower nutrient levels do indeed lead to decreased insulin levels. In the nematode worm Caenorhabditis elegans, mutation of the insulin/IGF1 receptor Daf-2 leads to a doubling of lifespan in a pathway dependent on the forkhead transcription factor Daf- $16,{ }^{15}$ and in flies similarly, mutation of chico, which encodes the insulin receptor substrate, is potent in increasing longevity. ${ }^{16}$ In flies, Foxo is not directly required but modulates the response to DR. ${ }^{17}$ Thus, inhibition of nutrient signaling, like dietary restriction, can increase lifespan. In worms, genetic analysis suggests that DR acts through a parallel pathway to the IIS pathway, although levels of Daf-2 activity impact on the effect of DR on longevity, ${ }^{18}$ suggesting some degree of overlap. In flies, the chico mutation and DR also appear to act through overlapping mechanisms because DR in chico $^{1}$ females had an adverse impact on longevity, suggesting that the chico mutation induces a state equivalent to DR. ${ }^{19}$

The IIS pathway relays signals to the nutrient-sensing complex TOR, a serine/threonine protein kinase that is an important nexus integrating responses to nutrients, growth factors, and stress as well as promoting cell growth, proliferation, or motility (Fig. 1; reviewed in ref. 20). Thus, TOR 
is likely to be the point linking the impact of rapamycin on longevity with that of dietary restriction.

The TOR protein (mTOR in mammals) is present in two distinct complexes, TORC1, which integrates nutrient signaling, and TORC2, which is involved in growth factor signaling and damping down stress responses. Each complex contains different partners, e.g., Raptor in TORC1 and Rector in TORC2, together with other accessory factors. High nutrient availability leads to a high adenosine diphosphate (ADP):adenosine monophosphate (AMP) ratio, resulting in lower AMP kinase signaling. If the ratio is shifted to high AMP on decreased nutrients, AMP kinase phosphorylates and activates the Tuberous Sclerosis Complex TSC1/2, which inhibits RHEB (Ras homolog enriched in brain), an upstream activator of TORC1 (Fig. 1), thus down-regulating TOR signaling on low nutrient levels. It is likely that calorie restriction acts at least in part through this pathway. Moreover, high nutrients lead to high circulating insulin levels and increased signaling through IIS. Immediately downstream of the insulin/IGF1 receptor is the insulin receptor substrate (IRS1, chico in flies), which in complex with phosphoinositide 3 (PI3) kinase leads to activation of AKT kinase and inhibition of TSC $1 / 2$, thus indirectly leading to activation of TORC1. Downstream targets of TORC1 phosphorylation include the ribosomal S6 kinase, translation initiation factor $4 \mathrm{E}$ binding protein (4EBP), and initiation factor eIF4B.

Phosphorylation of these components promotes ribosomemediated protein synthesis and possibly also results in increased glycolysis. Interestingly, TOR1 deletion in yeast increases oxygen consumption by the mitochondria ${ }^{21}$ and extends chronological lifespan. ${ }^{22}$ However, although an increase in density of components of the oxidative phosphorylation pathway are observed in tor $1 \Delta$ yeast, a decrease in mitochondrial membrane potential, possibly reflecting respiration uncoupling, is observed. ${ }^{23}$ By apparent contrast, rapamycin treatment of yeast cells is reported to result in a decrease in oxidative phosphorylation (OXPHOS) components, ${ }^{24}$ although this may be an effect of the time of addition. Whether such effects are also true for higher organisms (where aerobic rather than anaerobic cellular metabolism is the norm) remains to be determined, although hypoxia influences TOR activity through the hypoxia-inducible factor1 (HIF-1) factor. Metabolism is central to the importance of TOR in cells, and, in flies, inhibition of TOR signaling by loss-of-function mutation of various TOR pathway components (e.g., TOR $\left.{ }^{\mathrm{FRB}}, \mathrm{S} 6 \mathrm{~K} 1^{\mathrm{KQ}}\right)^{25}$ is only required in the fly fat body to achieve an increase in lifespan. ${ }^{25}$ By a strange quirk, rapamycin and newer analogs (rapalogs) are highly hydrophobic and thus likely to accumulate in fat. Perhaps part of rapamycin's impact on aging is mediated indirectly by suppressing inflammatory signals originating from fatty tissue; lower visceral fat deposition in CR individuals may obviate the need for rapamycin.

A common downstream effect of DR, decreased IIS or inhibition of TOR, is a decrease in protein synthesis. ${ }^{26}$ Therefore, it is unsurprising that chico ${ }^{1}$ mutant flies are dwarf and DR results in decreased body mass. Note that calorie restriction may not act entirely through the TOR pathway, because Sir2 histone deacetylase is up-regulated on calorie restriction in files and its overexpression results in lifespan extension that is not further increased by calorie restriction, suggesting that Sir2 acts in a CR longevity pathway. ${ }^{27}$ Thus, calorie restriction and rapamycin may overlap via TOR, but do not act through identical pathways (reviewed in ref. 28).

\section{Can lessons from calorie restriction and rapamycin be exploited to find an agent active in promoting longevity that protects against age-related decline?}

Importantly, and this is what makes the ITP study of direct relevance to possible human interventions, rapamycin was delivered as a component of the normal mouse diet, giving rise to the possibility of an "antiaging pill" for humans. However, rapamycin itself should not be the drug of choice given its known immunosuppressive effects and increased risk of some cancers. The lack of any detectable decrease in age-related disease in the rapamycin-treated mice provides a further caution against its use to delay aging in human populations. Calorie restriction, which does appear to delay onset of severe age-related morbidities in primates and also in humans, is not without its costs. Voluntary food deprivation to only $70 \%$ of ad libitum amounts would be very hard to maintain in a western culture with so much emphasis placed on enjoyment of food, in addition to other adverse effects (reviewed in ref. 10).

So what are the future prospects for developing an agent that can be used as a pill with the benefits of rapamycin and calorie restriction but with none of their drawbacks? Fortunately, a huge body of knowledge already exists on the TOR pathway, and rapamycin analogs (rapalogs) are already in development, particularly geared toward improved cancer chemotherapy (reviewed in ref. 29). However, extreme care in use of such agents is suggested by the exquisite feedback loops uncovered in early studies of rapalogs, where their effect was the opposite of that anticipated. Supposedly TORC1specific agents resulted in increased insulin signaling because TOR-activated S6K1 phosphorylates and destabilizes IRS1 and IRS2, thus damping down the insulin-signaling pathway (Fig. 1); TORC1 inhibition by rapalogs removes this negative feedback. Although rapamycin and newer rapalogs show high specificity for TORC1, the noncompetitive nature of inhibition leads to titration of mTOR away from TORC2 as the cell tries to produce more TORC1; the consequence is reduced AKT signaling. Thus, interfering with one aspect of TOR signaling may have unforeseen consequences on other aspects of cell physiology (reviewed in ref. 30).

Are other strategies in addition to rapalogs in sight? Yes: Inhibitors of MEK kinase and PI3 kinase block S6 kinase phosphorylation and prevent cell senescence. There is no additive effect with rapamycin, suggesting convergence on the TOR pathway. ${ }^{31}$ Pharmacological inhibition of the stress kinase p38MAPK also prevents cell senescence and restores proliferative potential to fibroblasts from patients with the progeroid Werner syndrome. ${ }^{32}$

What about other possible age-associated molecular pathways? Suppression of the tumor suppressor p53 in organisms such as flies that do not succumb to cancer leads to lifespan extension, ${ }^{33}$ whereas p53 overactivity results in premature aging of mice, ${ }^{34}$ at least in part by inducing expression of $\mathrm{p} 21^{\mathrm{CDKN} 1}$ required for maintaining cell cycle arrest in senescence. Interestingly, rapamycin, the drug that extends lifespan in mice, can delay senescence of cells in culture caused by ec- 
topic p21 expression. ${ }^{35}$ A further factor, Sir2 deacetylase, has been shown to be involved in lifespan extension and is activated by resveratrol. A very recent study suggests that Sir2dependent deacetylation and inactivation of the transcriptional activity of p53 may influence signaling that converges on TOR, as blocking p53 activity in flies correlates with decreased IIS activity in the fat body. ${ }^{36}$ Will all pharmacological agents active in promoting longevity converge on TOR to exert their effects? And if so, will it be possible to derive factors that promote healthy long life free of age-associated morbidity? By focussing on the pathways where calorie restriction and rapamycin meet, this may be a reasonable expectation.

The findings of Harrison et al. ${ }^{1}$ and Colman et al., ${ }^{2}$ while novel in terms of the species used, present pleasing validations of studies in model organisms such as yeast, worms, and flies using either TOR modulation or calorie restriction to extend lifespan (e.g., refs. 15, 22, 25, 37-39), suggesting that many aspects of aging in such model organisms may be directly applicable to human aging. The ITP and Wisconsin primate studies may thus serve to silence some of the critics of invertebrate aging research. More practically, the implication is that it will be valid to test effects of rapamycin analogs or other potential lifespan modulators in short-lived invertebrate species, avoiding use of vertebrates in early stages of drug validation and pathway elucidation. Such testing in lower organisms should allow rapid and large-scale screening of possible longevity-promoting agents, with obvious moral, ethical, and cost benefits.

\section{Acknowledgments}

I thank Drs. Penelope Mason and Charles Riddell for critical reading of the manuscript, and the BBSRC [BB/ E000924/1] and ESRC [RES-356-25-0016] for funding the work in my lab.

\section{Author Disclosure Statement}

Dr. Cox declares no competing financial or other interests.

\section{References}

1. Harrison DE, Strong R, Sharp ZD, Nelson JF, Castle CM, Flurkey K, Nadon NL, Wilkinson JE, Frenkel K, Carter CS, Pahor M, Javors MA, Fernandez E, Miller RA. Rapamycin fed late in life extends lifespan in genetically heterogeneous mice. Nature 2009;460:392-395.

2. Colman RJ, Anderson RM, Johnson SC, Kastman EK, Kosmatka KJ, Beasley TM, Allison DB, Cruzen C, Simmons HA, Kemnitz JW, Weindruch R. Caloric restriction delays disease onset and mortality in rhesus monkeys. Science 2009;325: 201-204.

3. Merry BJ. Effect of dietary restriction on lifespan. Rev Clin Gerontol1991;1:203-213.

4. Chai CK. Characteristics in inbred mouse populations plateaued by directional selection. Genetics 1966;54:743-753.

5. Neff M, Ledermann M, Saegesser H, Schneider V, Reichen $\mathrm{J}$. Low-dose oral rapamycin treatment reduces fibrogenesis, improves liver function, and prolongs survival in rats with established liver cirrhosis. J Hepatic 2006;45:786-796.

6. Colman RJ, Beasley TM, Allison DB, Weindruch R. Attenuation of sarcopenia by dietary restriction in rhesus monkeys. J Gerontol A Biol Sci Med Sci 2008;63:556-559.
7. Weindruch R, Walford RL. Dietary restriction in mice beginning at 1 year of age: effect on life-span and spontaneous cancer incidence. Science 1982;215:1415-1418.

8. Meyer TE, Kovacs SJ, Ehsani AA, Klein S, Holloszy JO, Fontana L. Long-term caloric restriction ameliorates the decline in diastolic function in humans. J Am Coll Cardiol 2006;47:398-402.

9. Fontana L, Klein S, Holloszy JO, Premachandra BN. Effect of long-term calorie restriction with adequate protein and micronutrients on thyroid hormones. J Clin Endocrinol Metab 2006;91:3232-3235.

10. Fontana L, Klein S. Aging, adiposity, and calorie restriction. JAMA 2007;297:986-994.

11. Villareal DT, Fontana L, Weiss EP, Racette SB, Steger-May $\mathrm{K}$, Schechtman KB, Klein S, Holloszy JO. Bone mineral density response to caloric restriction-induced weight loss or exercise-induced weight loss: A randomized controlled trial. Arch Intern Med 2006;166:2502-2510.

12. Desai MM, Zhang P, Hennessy CH. Surveillance for morbidity and mortality among older adults-United States, 1995-1996. MMWR CDC Surveill Summ 1999;48:7-25.

13. Butcher SK, Killampalli V, Lascelles D, Wang K, Alpar EK, Lord JM. Raised cortisol:DHEAS ratios in the elderly after injury: Potential impact upon neutrophil function and immunity. Aging Cell 2005;4:319-324.

14. Bishop NA, Guarente L. Genetic links between diet and lifespan: Shared mechanisms from yeast to humans. Nat Rev Genet 2007;8:835-844.

15. Kenyon C, Chang J, Gensch E, Rudner A, Tabtiang R. A C. elegans mutant that lives twice as long as wild type. Nature 1993;366:461-464.

16. Clancy DJ, Gems D, Harshman LG, Oldham S, Stocker H, Hafen E, Leevers SJ, Partridge L. Extension of life-span by loss of CHICO, a Drosophila insulin receptor substrate protein. Science 2001;292:104-106.

17. Giannakou ME, Goss M, Partridge L. Role of dFOXO in lifespan extension by dietary restriction in Drosophila melanogaster: not required, but its activity modulates the response. Aging Cell 2008;7:187-198.

18. Iser WB, Wolkow CA. DAF-2/insulin-like signaling in C. elegans modifies effects of dietary restriction and nutrient stress on aging, stress and growth. PLoS One 2007;2: e1240.

19. Clancy DJ, Gems D, Hafen E, Leevers SJ, Partridge L. Dietary restriction in long-lived dwarf flies. Science 2002; 296:319.

20. Wullschleger S, Loewith R, Hall MN. TOR signaling in growth and metabolism. Cell 2006;124:471-484.

21. Bonawitz ND, Chatenay-Lapointe M, Pan Y, Shadel GS. Reduced TOR signaling extends chronological life span via increased respiration and upregulation of mitochondrial gene expression. Cell Metab 2007;5:265-277.

22. Kaeberlein M, Powers RW 3rd, Steffen KK, Westman EA, $\mathrm{Hu}$ D, Dang N, Kerr EO, Kirkland KT, Fields S, Kennedy BK. Regulation of yeast replicative life span by TOR and Sch9 in response to nutrients. Science 2005;310:1193-1196.

23. Pan Y, Shadel GS. Extension of chronological life span by reduced TOR signaling requires down-regulation of Sch9p and involves increased mitochondrial OXPHOS complex density. Aging 2009;1:131-145.

24. Bandhakavi S, Xie H, O'Callaghan B, Sakurai H, Kim DH, Griffin TJ. Hsf1 activation inhibits rapamycin resistance and TOR signaling in yeast revealed by combined proteomic and genetic analysis. PLoS One 2008;3:e1598. 
25. Kapahi P, Zid BM, Harper T, Koslover D, Sapin V, Benzer S. Regulation of lifespan in Drosophila by modulation of genes in the TOR signaling pathway. Curr Biol 2004;14:885890.

26. Proud CG. Regulation of protein synthesis by insulin. Biochem Soc Trans 2006;34(Pt 2):213-216.

27. Rogina B, Helfand SL. Sir2 mediates longevity in the fly through a pathway related to calorie restriction. Proc Natl Acad Sci USA 2004;101:15998-16003.

28. Guarente L. Calorie restriction and SIR2 genes-towards a mechanism. Mech Ageing Dev 2005;126:923-928.

29. Abraham RT, Gibbons JJ. The mammalian target of rapamycin signaling pathway: Twists and turns in the road to cancer therapy. Clin Cancer Res 2007;13:3109-3114.

30. Sabatini DM. mTOR and cancer: insights into a complex relationship. Nat Rev Cancer 2006;6:729-734.

31. Demidenko ZN, Shtutman M, Blagosklonny MV. Pharmacologic inhibition of MEK and PI-3K converges on the mTOR/S6 pathway to decelerate cellular senescence. Cell Cycle 2009;8:1896-1900.

32. Davis T, Baird DM, Haughton MF, Jones CJ, Kipling D. Prevention of accelerated cell aging in Werner syndrome using a p38 mitogen-activated protein kinase inhibitor. J Gerontol A Biol Sci Med Sci 2005;60:1386-1393.

33. Donehower LA. Longevity regulation in flies: A role for p53. Aging 2009;16-8.

34. Tyner SD, Venkatachalam S, Choi J, Jones S, Ghebranious N, Igelmann H, Lu X, Soron G, Cooper B, Brayton C, Hee Park S, Thompson T, Karsenty G, Bradley A, Donehower LA. p53 mutant mice that display early ageing-associated phenotypes. Nature 2002;415:45-53.
35. Demidenko ZN, Zubova SG, Bukreeva EI, Pospelov VA, Pospelova TV, Blagosklonny MV. Rapamycin decelerates cellular senescence. Cell Cycle 2009;8:1888-1895.

36. Bauer JH, Morris SNS, Chang C, Flatt T, Wood JG, Helfand SL. dSir2 and Dmp53 interact to mediate aspects of CR-dependent life span extension in D. melanogaster. Aging 2009;1:38-48.

37. Powers RW 3rd, Kaeberlein M, Caldwell SD, Kennedy BK, Fields S. Extension of chronological life span in yeast by decreased TOR pathway signaling. Genes Dev 2006;20: 174-184.

38. Vellai T, Takacs-Vellai K, Zhang Y, Kovacs AL, Orosz L, Muller F. Genetics: Influence of TOR kinase on lifespan in C. elegans. Nature 2003;426:620.

39. Jia K, Chen D, Riddle DL. The TOR pathway interacts with the insulin signaling pathway to regulate C. elegans larval development, metabolism and life span. Development 2004;131:3897-3906.

Address correspondence to: Lynne S. Cox Department of Biochemistry University of Oxford South Parks Road Oxford OX1 3QU United Kingdom

E-mail: lynne.cox@bioch.ox.ac.uk

Received: July 24, 2009 Accepted: July 25, 2009 